\title{
Modified Planar Inverted F Antenna for Wimax Application
}

\author{
S.Gopalakrishnan ${ }^{1}$, R.Pradeep ${ }^{1}$, P.Ramya ${ }^{2}$ \\ UG Scholars, Bannari Amman Institute of Technology, Sathyamangalam ${ }^{1}$ \\ Assistant Professor, Bannari Amman Institute of Technology, Sathyamangalam²
}

\begin{abstract}
In this paper the PIFA antenna with strips extended from ground plane is proposed for the WIMAX application. The PIFA antenna works at the frequency of $3.5 \mathrm{GHz}$ with the satisfying antenna parameters like directivity, gain and radiated power. The figure 8 type radiation pattern is obtained as the result. The project simulation is carried out in Agilent Technologies Advanced Design System (ADS).
\end{abstract}

Keywords- ADS, PIFA, FR4, SAR, WIMAX

\section{INTRODUCTION}

The planar inverted F antenna is popular for portable Interaction of handset antennas with human body is and wireless devices because of its low profile,size is small length of handset boxes used in are considerably a great and low SAR value. The other major advantages are it is consideration in cellular communications.

easy to fabricate, it is off low cost, and also its structure is simple. Also, PIFA's inherent bandwidth is higher than the bandwidth of the conventional patch antenna.

The basic PIFA (a "grounded" patch antenna - $\lambda / 4$ patch length instead of the conventional $\lambda / 2$ ) consists of a top plate element, a feed wire feeding the resonating top plate,a ground plane and a DC-shorting plate that is connecting the ground and the top plate at one end of the resonating patch.

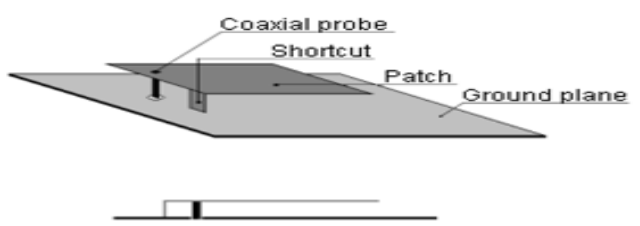

Fig 1: General PIFA antenna

The classical PIFA design techniques are used to make a quarter-wavelength resonant patch for the required resonant frequency while the height of the top plate above the ground plane is fixed $(\approx 0.04 \lambda)$. The location of the shorting plate and feeding point is optimized in order to achieve the best matching.

Another type of patch antenna is the Planar Inverted-F Antenna (PIFA) common in mobile phones with built-in antennas.(The Planar Inverted-F antenna (PIFA) is increasingly used in the cellular phones.

The resonant of the antenna is a quarter-wavelength and it has good Specific Absorption Rate(SAR) properties. The shape of the antenna resembles an inverted $F$ and so the name PIFA. The Planar Inverted-F Antenna is popular because it has a low profile and an omnidirectional pattern.
The greater than those used today. SAR and temperature rise user's body, especially head and hand, influence on the in a human head have been calculated.

Single band helical and side radiation patterns have been studied. Furthermore, thermal effect caused due to PIFA antenna on the tissues exposed to unlimited electromagnetic energy are studied.

The heat produced by the PIFA antenna is less compared to the other antennas and also the SAR value is also less when compared to other antennas.

\section{ANTENNA DESIGN}

The geometry of the proposed antenna shown in figure 2 is fabricated on an FR4 substrate with thickness of $3.2 \mathrm{~mm}$, relative permittivity of 4.6 .

The overall dimensions $43.2 \mathrm{~mm} \times 12.7 \mathrm{~mm}$ (LI x WI) contain an antenna portion $10 \mathrm{~mm} \times 12.7 \mathrm{~mm}$ (LIO x WI) and a ground plane $33.2 \mathrm{~mm} \times 10 \mathrm{~mm}$. The parameters and dimensions are shown in Fig. 1(c) and listed in Table 1, respectively.

A PIFA type antenna printed on outer side of antenna portion excites the $3.5 \mathrm{GHz}$ resonant mode with the satisfying antenna parameters.

The antenna is built on the FR4 substrate of the thickness $3.2 \mathrm{~mm}$. The effective dielectric constant of the substrate is 4.6 .

The FR4 substrate is used to be very cost effective and easy to fabricate the antenna. Hence the FR4 substrate is widely preferred than any other substrate. 


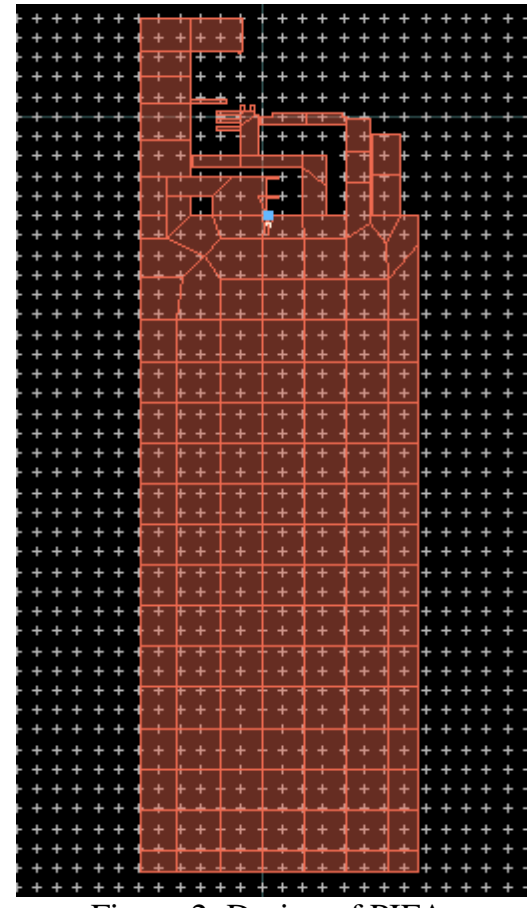

Figure 2: Design of PIFA

The dimensions of the strip is given as

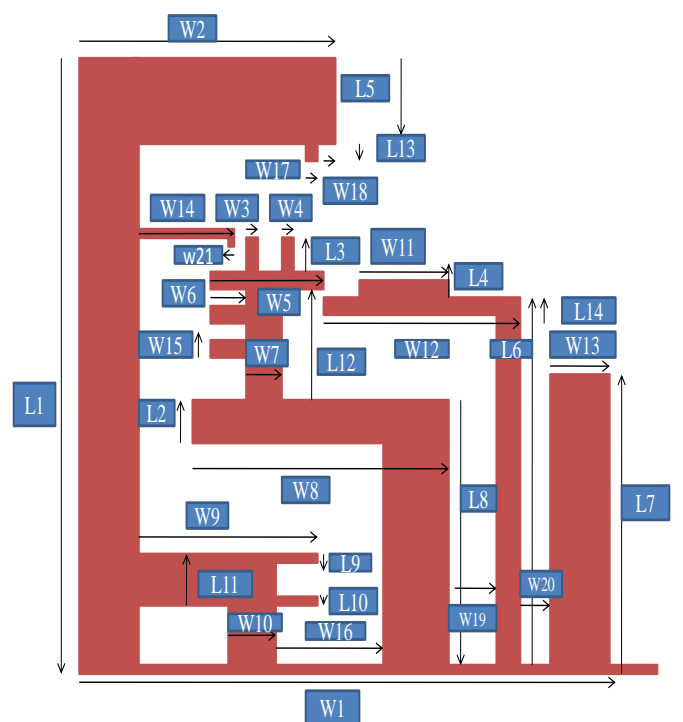

Figure 3: Dimensions of the strips

The dimensions of the entire antenna is shown in the tabulation below

Table 1: Dimensions

\begin{tabular}{|l|l|l|l|l|l|l|l|}
\hline L1 & L2 & L3 & L4 & L5 & L6 & L7 & L8 \\
\hline 10 & 0.6 & 0.3 & 0.2 & 1.7 & 4.9 & 4.1 & 3 \\
\hline L9 & L10 & L11 & L12 & L13 & L14 & W1 & W2 \\
\hline 0.1 & 0.1 & 2 & 2.1 & 0.1 & 0.1 & 5 & 0.2 \\
\hline W3 & W4 & W5 & W6 & W7 & W8 & W9 & W10 \\
\hline 0.4 & 2 & 1.2 & 0.8 & 6.6 & 4.3 & 4.3 & 3.4 \\
\hline W11 & W12 & W13 & W14 & W15 & W16 & W17 & W18 \\
\hline 5.4 & 1.4 & 2 & 0.2 & 0.2 & 1.8 & 0.1 & 0.1 \\
\hline W19 & W20 & W21 & & & & & \\
\hline 0.9 & 0.1 & 0.2 & & & & & \\
\hline
\end{tabular}

The Planar Inverted $\mathrm{F}$ antenna has Low rate of SAR absorption. SAR is defined as the specific absorption rate which means the amount of radiation absorbed by the human tissue whenever it was exposed to the radiation. PIFA has the reduced backward direction radiation and it is a low profile antenna. The PIFA has Omni directional patterns and it can maintain the high to moderate amount of gain whenever it was subjected to both horizontal and vertical polarization.

\section{RESULTS}

The antenna parameters that affect the resonant modes are discussed in this paragraph. It shows the simulated S 11 of the proposed antenna which works at the frequency of 3.5 $\mathrm{GHz}$ of WIMAX application.

The simulation is carried out in Advanced Design System (ADS). It produces the 8 pattern shape radiation to its orthogonal side of each lobe.

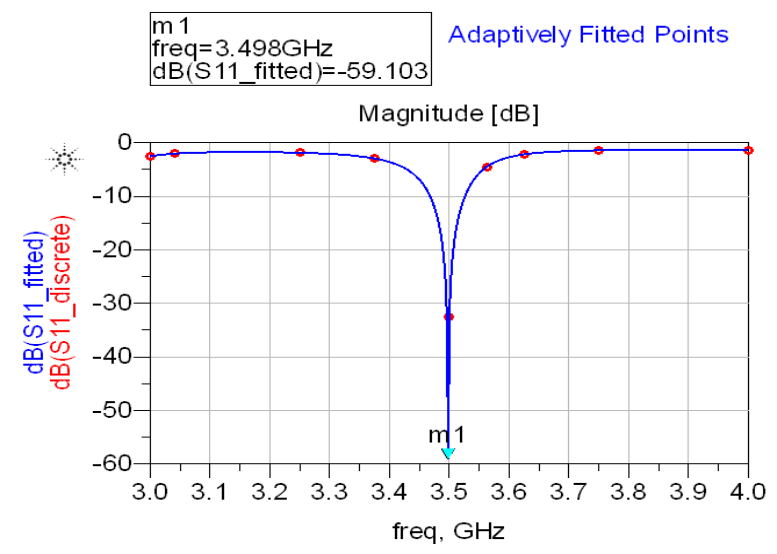

Figure 4: Obtained return loss at $\mathrm{S}(1,1)$

The return loss at the port $\mathrm{S}(1,1)$ is obtained at the frequency of $3.5 \mathrm{GHz}$ frequency of WIMAX application.

The radiation patterns that are obtained at this particular frequency is shown below

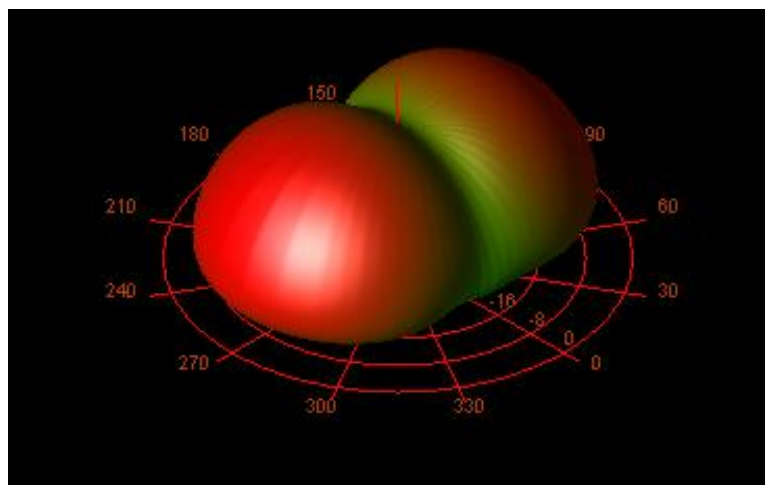

Figure 5: 3D Radiation pattern

The antenna parameters that are obtained during the simulation was at satisfactory level. The parameters are given below 


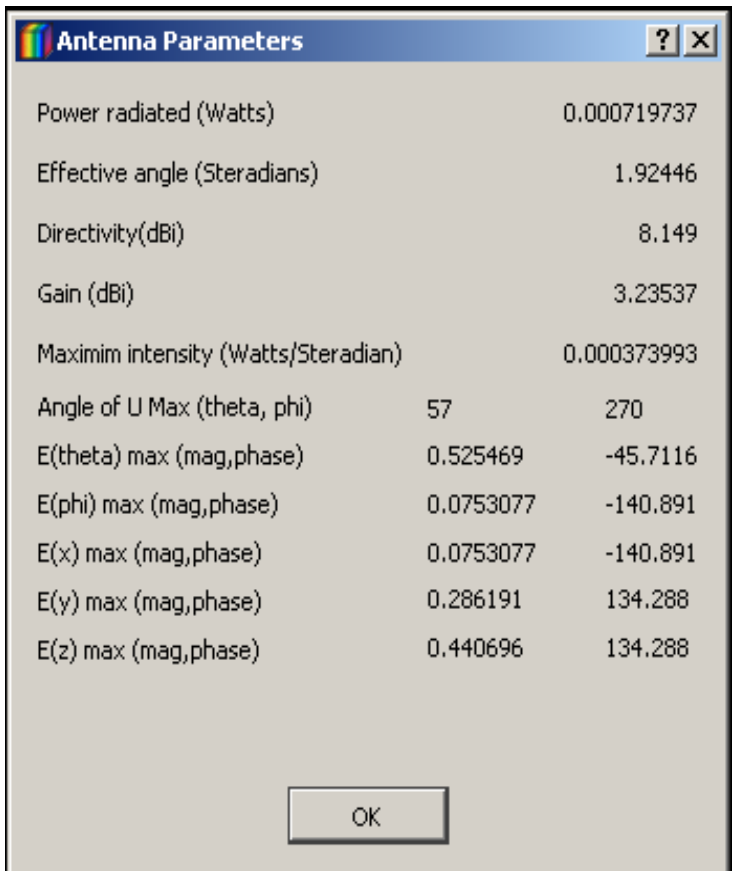

Figure 6: Antenna Parameters

The radiation throughout the antenna is shown here. The red color denoted the maximum radiation across the antenna.

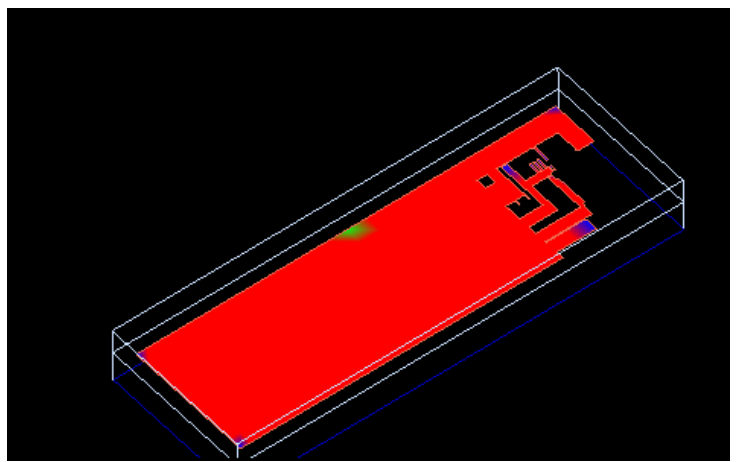

Figure 7: Radiating region

The $2 \mathrm{D}$ radiation pattern is also obtained which also denotes the efficiency of the antenna in percent. Hence the radiation pattern is given below

\section{Power}

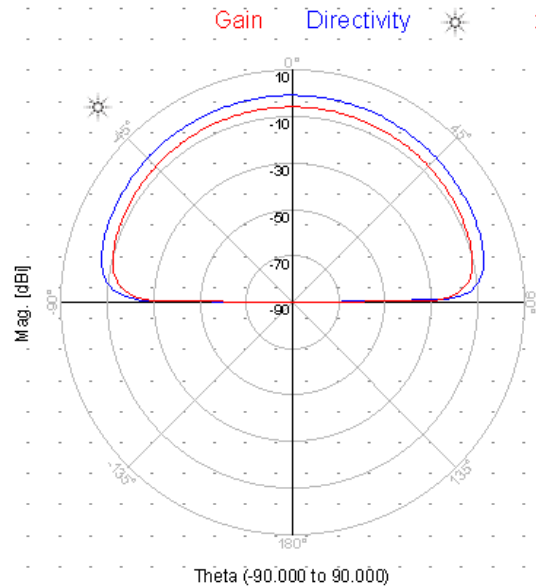

Figure 8: 2D radiation pattern

\section{CONCLUSION}

The $2 \mathrm{D}$ radiation pattern shows that the antenna has achieved the satisfactory level of radiating properties and antenna parameters. The directivity of the antenna is 8.149 and the gain of the antenna is 3.23537. The proposed method uses FR4 as substrate and hence the cost of the antenna is low.

\section{REFERENCE}

1. Iftikhar Ahmed, Imran Shoaib, NosherwanShoaib, ArslanRasheed and Sultan Shoaib"A Printed Hybrid Loop Planar Inverted-F Antenna for Next Generation Handheld Terminals" $7^{\text {th }}$ European Conference on Antenna and Propagation 2013

2. Jimmy Tsai, Yih-Chien Chen, and Chung-Yen Wu "Dual-Band Planar Inverted-F Antenna for Application in WIMAX,UNII,HIPERLAN and ISM" Proceedings of Asia Pacific Microwave Conference 2012, Kaohsiung, Taiwan, Dec. 4-7, 2012

3. SungHyun Kim, HyunWook Moon, WooJoong Kim, JaeSik Kim, and YoungJoong Yoon "A Compact GPS and WLAN PIFA for Full Metal-Rimmed Mobile Handset Using the Ground Bridges" Proceedings ofAsia-Pacific Microwave Conference 2013

4. Wen-Shan Chen, Bau-Yi Lee, Jing-Ju Lu, Ke-Ming Lin ,Ching-Yu Huang and Chih-Kai Yang "A PIFA Type USB Dongle Antenna for WLAN Applications" CSQRWC 2013

5. H. M. Salgado and L. Qi, J. R. Pereira, "Fractal Monopole Antenna for WLAN USB Dongle," 2009 Loughborough Antennas \&Propagation Conference.

6. S. H. Lee and Y. Sung, "Multiband Antenna for Wireless USB Dongle Applications," IEEE Antennas Wireless Propag. Lett., vol. 10, pp. 25-28, 2011

7. C. C. Lin, S. W. Kuo, and H. R. Chuang,"A $3.5-\mathrm{GHz}$ Printed MeanderLineAntenna for USB WLAN with Notebook-PC Housing," IEEE Microwave and Wireless Components Lett., vol. 15 , pp. 546-548,2005.

8. D. Kim, U. Kim, and 1. Choi, "Design of a Wideband Internal Monopole Antenna for Wireless USB dongle application,"Proceedings of AsiaPacificMicrowave Conference 2010.

9. W. C. Liu and Y. L. Chen, "Compact strip-monopole antenna for WLAN-band USB dongle application," Electronics Letters, vol. 47, 2011.

10. PIFA based Reconfigurable Multiband Antenna for Wireless Applications Hattan F. AbuTarboush1 , R. Nilavalan and T. Peter.

11. Study of Planar Inverted F-shaped (PIFA) Antenna in WIMAX Band Akanksha khare, Paurush bhulania.

12. Small Size Planar Inverted-F Antenna For WiMAX applications Sunakshi Luthra1 and Kiranpreet Kaur. 